NEAR INFRARED REFLECTANCE SPECTROSCOPY AS A PROCESS SIGNATURE IN URANIUM OXIDES

J. W. Plaue, G. L. Klunder, K. R. Czerwinski, I. D. Hutcheon

March 21, 2012

Methods \& Applications of Radianalytical Chemistry Kona, HI, United States

March 25, 2012 through March 30, 2012 
This document was prepared as an account of work sponsored by an agency of the United States government. Neither the United States government nor Lawrence Livermore National Security, LLC, nor any of their employees makes any warranty, expressed or implied, or assumes any legal liability or responsibility for the accuracy, completeness, or usefulness of any information, apparatus, product, or process disclosed, or represents that its use would not infringe privately owned rights. Reference herein to any specific commercial product, process, or service by trade name, trademark, manufacturer, or otherwise does not necessarily constitute or imply its endorsement, recommendation, or favoring by the United States government or Lawrence Livermore National Security, LLC. The views and opinions of authors expressed herein do not necessarily state or reflect those of the United States government or Lawrence Livermore National Security, LLC, and shall not be used for advertising or product endorsement purposes. 
LOG \# 263

\title{
NEAR INFRARED REFLECTANCE SPECTROSCOPY AS A PROCESS SIGNATURE IN URANIUM OXIDES
}

\author{
JW Plaue $(1,2,3)$ \\ plaue2@1lnl.gov \\ 7000 East Ave, L-293 \\ Livermore, CA 94551 \\ GL Klunder (2) \\ klunder1@1lnl.gov \\ 7000 East Ave, L-091 \\ Livermore, CA 94551 \\ ID Hutcheon (2) \\ hutcheon1@1lnl.gov \\ 7000 East Ave, L-231 \\ Livermore, CA 94551 \\ KR Czerwinski (1) \\ czerwin2@unlv.nevada.edu \\ University of Nevada, Las Vegas \\ 4505 Maryland Parkway, Box 454003
}

(1) Radiochemistry Program, Department of Chemistry, University of Nevada, Las Vegas

(2) Chemical Sciences Division, Lawrence Livermore National Laboratory

(3) Defense Nuclear Facilities Safety Board 


\title{
NEAR INFRARED REFLECTANCE SPECTROSCOPY AS A \\ PROCESS SIGNATURE IN URANIUM OXIDES
}

\author{
JW Plaue ${ }^{1,2,3}$; GL Klunder ${ }^{2}$; ID Hutcheon ${ }^{2} ;$ KR Czerwinski $^{1}$ \\ (1) Radiochemistry Program, Department of Chemistry, University of Nevada, Las Vegas \\ (2) Chemical Sciences Division, Lawrence Livermore National Laboratory \\ (3) Defense Nuclear Facilities Safety Board
}

\begin{abstract}
Near-infrared (NIR) reflectance spectroscopy was examined as a potential tool for the determination of forensic signatures indicative of the chemical process history of uranium oxides. The ability to determine the process history of nuclear materials is a desired, but underdeveloped, area of technical nuclear forensics.

Application of the NIR technique potentially offers a quick and non-destructive tool to serve this need; however, few data have been published on the compounds of interest. The viability of NIR was investigated through the analysis of a combination of laboratory-derived and real-world uranium precipitates and oxides. A set of reference uranium materials was synthesized in the laboratory using the commonly encountered aqueous precipitation reactions for uranium ore concentration and chemical separation processes (ammonia, hydrogen peroxide, sodium hydroxide, ammonium carbonate, and magnesia). NIR spectra were taken on a range of samples heat treated in air between $85-750{ }^{\circ} \mathrm{C}$. X-ray diffraction patterns were also obtained to complement the NIR analysis with crystal phase information. Similar analyses were performed using a set of real-world samples, with process information obtained from the literature, to provide a comparison between materials synthesized in the laboratory and samples representative of industrial processes.
\end{abstract}

Keywords: nuclear forensics, uranium oxide, uranium ore concentrate, yellowcake, near-infrared reflectance spectroscopy, NIR

\section{Introduction}

Technical nuclear forensics has emerged as a key discipline to provide law enforcement entities with characterization and interpretations regarding the age, composition, provenance, industrial history, and implications of nuclear materials [1]. To-date, much effort has focused on techniques, such as trace element and isotopic analysis, which have demonstrated the ability to link material to a likely geological source [2] [3]. Considerably less effort has been put forth in developing techniques to determine the chemical process history of nuclear materialsalso a desirable outcome of technical nuclear forensics. For example, information on the chemical process history, 
termed process signatures, can be useful in combination with observations of industrial chemical usage to establish potential manufacturing origin.

An obvious starting point for research into process signatures is the precipitation history of uranium oxides due the ubiquity of uranium in the nuclear fuel cycle and the strong likelihood that a given sample was precipitated from solution at some point in its industrial lifecycle. Near infrared (NIR) spectroscopy offers the potential for rapid and non-contact material analysis without the need for sample preparation - a benefit of particular interest when working with radioactive materials. NIR analysis has been previously applied for industrial process control and forensic-like applications [4]; however, no work has examined NIR as a tool for nuclear forensics or more broadly examined the NIR spectra of the uranium precipitants and oxides examined in this paper. This work explored two objectives on whether NIR spectra: (1) can be utilized to distinguish common uranium precipitates from each other and (2) further distinguish features in precipitates that have been decomposed into oxides through heat treatments (i.e., calcination).

The first objective can be accomplished through other means; however, these methods require extensive sample preparation and time to perform the analyses. As described by Hausen, historically a combination of x-ray diffraction (XRD), infrared spectroscopy, and differential thermal analysis was applied in the analysis of yellowcakes [5]. Recently, Varga demonstrated the ability to utilize infrared spectra to identify and classify uranium ore concentrates for nuclear forensics applications [6].

The second objective has not been demonstrated for precipitants involving volatile anions (ammonium hydroxide, hydrogen peroxide, or ammonium carbonate). The volatile nature of these reagents means that residual forms are unlikely to persist after exposure to elevated temperatures; however, work by these authors has observed visually distinct morphologies in uranium oxides resulting from different precipitation agents. In a wide number of cases, the NIR technique has been successfully shown to distinguish samples with different particle size and morphology, which suggested the potential utility for discerning process origins in uranium oxides of different morphologies.

A note on historical terminology - the term 'yellowcake' is overly generic and can be used to describe both the precipitates of uranium in the ore refining process and the oxides resulting from subsequent heat treatment. Collectively, these precipitates and oxides are termed uranium ore concentrates; however, this term is also misleading in that these materials are commonly utilized in steps of the fuel cycle beyond ore refinement. All of 
these materials are described in this work as uranium precipitates and oxides to accurately reflect their presence in the manufacturing and recycling of nuclear fuel, as well as weapons applications.

\section{EXPERIMENTAL}

\section{Preparation of laboratory-derived uranium oxides and collection of known real-world materials}

Uranium is commonly precipitated from aqueous acidic solutions using one of five reagents: ammonium hydroxide, hydrogen peroxide, ammonium carbonate, magnesia, and sodium hydroxide. Samples of uranium precipitates were prepared utilizing each of these reagents (1.0 M uranyl nitrate with $1.0 \mathrm{M}$ reagent, except for magnesia which was used as 15 weight percent slurry). The precipitates were heated in air for 12 hours at temperatures of $85,150,400,600$, and $750{ }^{\circ} \mathrm{C}$. In addition to the NIR measurements, chemical phase information was obtained using powder x-ray diffraction (XRD) pattern matching against the 2009 database issued by the International Centre for Diffraction Data ${ }^{\circledR}$ using the EVA software package by Bruker.

Samples of real-world uranium precipitates and oxides, primarily as uranium ore concentrates, were obtained from laboratory archives and subjected to the same NIR and XRD analysis procedures outlined previously. The literature was used to identify the precipitating agent and any available information on thermal treatments.

\section{NIR measurements and data analysis}

NIR spectra were acquired using an Analytical Spectral Devices Inc. Labspec Pro spectrometer with three detectors spanning the spectral range from 350-1000, 1000-1800, and 1800-2500 nm. Fiber optic bundles were used to transmit light from a $20 \mathrm{~W}$ tungsten halogen source to and from the sample. Spectra were scanned in $1 \mathrm{~nm}$ steps with a spectral resolution of approximately $10 \mathrm{~nm}$. The samples required no preparation and 10 spectra were acquired for each sample in about 1 second.

Spectral processing was accomplished using The Unscrambler ${ }^{\circledR}$ software package, version 10.1 (CAMO Inc, Oslo Norway). Raw spectra were preprocessed and presented using the standard normal variate to minimize baseline shifts and multiplicative interferences of particle size as recommended by Barnes [7].

\section{RESULTS AND DISCUSSION}

\section{NIR spectral features can distinguish precipitates}

NIR spectra were taken for all laboratory-derived samples and are presented in Figure 1 along with chemical phase information obtained through XRD pattern matching. The two NIR regions are presented separately since different detectors are used in each region. Peak assignments for $\mathrm{O}-\mathrm{H}, \mathrm{N}-\mathrm{H}$, and other groups are indicated in the figure and are discussed later. Each uranium phase resulted in a unique spectrum, sufficient to distinguish the 
origin of a given sample by visual evaluation of the spectra. Figure 2 compares spectra for each of the precipitates heated to $85^{\circ} \mathrm{C}$ in the first NIR detector region. Note that common absorption wavelengths were shared by several samples; however, the relative ratios of these absorption peaks were unique and sufficient to make distinctions.

As process temperatures were increased, spectral changes occurred consistent with the change in chemical species. The chemical species can be grouped by the volatility of their precipitants, resulting in a volatile set (ammonium hydroxide, hydrogen peroxide, and ammonium carbonate) and a non-volatile set (sodium hydroxide and magnesia). At elevated temperatures of $400{ }^{\circ} \mathrm{C}$ to $750{ }^{\circ} \mathrm{C}$, the volatile components of the precipitates are liberated and the uranium begins converging to a triuranium octoxide $\left(\mathrm{U}_{3} \mathrm{O}_{8}\right)$. The NIR spectra were nearly identical for each of the $\mathrm{U}_{3} \mathrm{O}_{8}$ samples with dominant absorptions at 1505 and $1565 \mathrm{~nm}$. This result suggests that NIR is insufficiently sensitive to morphological differences to reliably distinguish between chemically identical species derived through different routes.

\section{Analysis of spectral features}

The complexity of the NIR spectral region can make specific assignment of features difficult; however, absorption bands were assigned corresponding to the functional groups of the various uranium compounds using available information and simple experiments. The literature on NIR spectroscopy of uranium compounds is very limited, with most of the published information authored by Frost and focused on the examination of mineral phases including, calcium uranyl phosphates (autunites ) [8], uranyl arsenates [9], uranyl selenites (haynesite) [9], uranyl carbonates (rutherfordines) [10], uranyl selenites (haynesite) [11], and copper uranyl phosphates (torbernites and metatorbernites) [12]. No spectral similarities were observed between our laboratory-derived samples and these mineral phases, despite the analogous functionalities of the carbonate group (observed by Frost at 1650, 1700, and $1750 \mathrm{~nm}$ ), and the uranyl group (observed by Frost at 1060 and 1144, nm). Hanchar [13] also assigned uranyl to a broad absorption at $714 \mathrm{~nm}$, which was not observed in any of these minerals.

In general, NIR spectral features are primarily attributable to the overtone and combination bands of $\mathrm{C}-\mathrm{H}$, $\mathrm{O}-\mathrm{H}$, and $\mathrm{N}-\mathrm{H}$ bonds [14]. In addition, there are some absorptions that arise from crystal field effects and electronic transition in the actinides [15]. In Figures 1 and 2, many of the low temperature samples, particularly the $85^{\circ} \mathrm{C}$ set, exhibit characteristic absorption peaks for the water O-H bonds at 1450 and $1940 \mathrm{~nm}$, which arise from the first overtone and combination of asymmetric stretching and bending, respectively. Similarly, the overtone of the N-H stretch near $1480 \mathrm{~nm}$ and the stretch and bend combinations at 2050 and $2150 \mathrm{~nm}$ were observed in the ammonium hydroxide and ammonium carbonate derived precipitates. Other overtones for the functional groups expected based 
on the species were not observed, including carbonate $(2550$ and $2350 \mathrm{~nm})$ and peroxide $(2060 \mathrm{~nm}$ for aqueous species). The absorptions near 1505 and $1565 \mathrm{~nm}$ observed in the $\mathrm{U}_{3} \mathrm{O}_{8}$ samples do not appear to be overtones as there are no corresponding absorptions in the higher wavelength region typically associated with combination bands, thus these absorptions are likely the result of crystal field effects or electronic transitions.

Analyses of pure solid reagents (magnesia and ammonium carbonate) were also performed for comparative peak assignments. For magnesia, the spectra demonstrate a unique absorption at $1395 \mathrm{~nm}$, which was found to be present in all but the $750{ }^{\circ} \mathrm{C}$ synthetic sample. For ammonium carbonate, absorptions were observed at 1640, 1975, $2040,2175 \mathrm{~nm}$, of which the $2040 \mathrm{~nm}$ peak was found for the $85^{\circ} \mathrm{C}$ ammonium uranyl carbonate synthetic samples.

Edison [16] and Varga [6] provide infrared analysis of some relevant uranium ore concentrates where the O-H and N-H fundamentals around 2900 and $3185 \mathrm{~nm}$ were identified, respectively. Corresponding first fundamental overtones would appear around 1440 and $1580 \mathrm{~nm}$, of which the $1440 \mathrm{~nm}$ absorption was observed for all samples and the $1580 \mathrm{~nm}$ absorption nearly observed with the ammonium carbonate $(1585 \mathrm{~nm})$ and ammonium hydroxide samples $(1570 \mathrm{~nm})$. The other fundamental infrared spectrum features observed by these authors occur at wavelengths unlikely to result in features detectable in the NIR region.

\section{Classification of real-world samples}

As an example of the utility of the NIR methodology, Figure 3 shows comparisons of spectra between the laboratory-derived and real-world samples with similar known process history. Precipitates of hydrogen peroxide (Figure 3a) and ammonium hydroxide (Figure 3b) have good agreement in spectral patterns, which is consistent with XRD results indicating that all samples are metastudites for the peroxide precipitates and $\mathrm{U}_{3} \mathrm{O}_{8}$ for ammonium hydroxide precipitates heated to elevated temperatures. The variability in the spectra from the real world samples indicates the potential to discriminate samples from different sources due to processing differences, source material or contaminants. Similar results were observed with the other precipitates; particularly good ammonium carbonate and slightly less so for magnesia and sodium hydroxide. Current work is investigating classification and grouping of these samples based on process with comparisons to real world samples using principal component analysis.

\section{CONCLUSIONS}

This paper presented the first NIR spectra for common uranium precipitates and resulting oxides and demonstrated the technique as a rapid, non-contact, non-destructive method of analysis. The NIR spectra were sufficiently unique for each compound to distinguish origin through visual examination. Assignments of absorption peaks were made for common $\mathrm{O}-\mathrm{H}$ and $\mathrm{N}-\mathrm{H}$ combination and overtone bands, in addition to several features 
associated with $\mathrm{U}_{3} \mathrm{O}_{8}$, magnesia, and sodium oxide. Continuing efforts are investigating definitive band assignments for other absorption features. Comparisons between laboratory-derived and real-world samples were good and suggested that the utility of NIR for accurate classification of unknown samples may be achieved using multivariate methods such as principal component analysis. NIR was unable to distinguish between the same chemical phases (e.g., $\mathrm{U}_{3} \mathrm{O}_{8}$ ) derived through different precipitation methods despite differences in sample morphology. With additional refinement, NIR should provide an important tool for nuclear forensic analysis of uranium precipitates and oxide and likely other actinides.

\section{ACKNOWLEDGEMENTS}

This work performed under the auspices of the U.S. Department of Energy by Lawrence Livermore National Laboratory under Contract DE-AC52-07NA27344. The views and opinions of authors expressed herein do not necessarily state or reflect those of the United States government, the Defense Nuclear Facilities Safety Board, or Lawrence Livermore National Security, LLC, and shall not be used for advertising or product endorsement purposes.

The authors wish to thank the Defense Nuclear Facilities Safety Board for support of continuing education and the LLNL Laboratory Directed Research and Development program (10-SI-016) for programmatic funding.

\section{REFERENCES}

[1] U.S. Joint Working Group of the American Physical Society and the American Association for the Advancement of Science, Nuclear Forensics: Role, State of the Art, and Program Needs, 2008.

[2] Z. Varga, M. Wallenius, K. Mayer, E. Keegan, S. Millet, Anal. Chem. 81 (2009) 8327-8334.

[3] K. Mayer, M. Wallenius, I. Ray, Analyst 130 (2005) 433.

[4] D.A. Burns, E.W. Ciurczak, Handbook of Near-Infrared Analysis, 3rd ed., Marcel Dekker, New York, NY, 2007.

[5] D.M. Hausen, Journal of the Minerals, Metals and Materials Society 50 (1998) 45-47.

[6] Z. Varga, B. Öztürk, M. Meppen, K. Mayer, M. Wallenius, C. Apostolidis, Radiochimica Acta (2011) 110822022707002 .

[7] R.J. Barnes, M.S. Dhanoa, S.J. Lister, Applied Spectroscopy 43 (1989) 772-777.

[8] R.L. Frost, K.L. Erickson, M.O. Adebajo, M.L. Weier, Spectrochimica Acta Part A: Molecular and Biomolecular Spectroscopy 61 (2005) 367-372.

[9] R.L. Frost, O. Carmody, K.L. Erickson, M.L. Weier, Spectrochimica Acta Part A: Molecular and Biomolecular Spectroscopy 61 (2005) 1923-1927.

[10] R.L. Frost, J. Čejka, Journal of Raman Spectroscopy 40 (2009) 1096-1103.

[11] R.L. Frost, J. Cejka, Spectrochimica Acta Part A: Molecular and Biomolecular Spectroscopy 71 (2009) 1959-1963.

[12] R.L. Frost, K.L. Erickson, O. Carmody, M.L. Weier, Spectrochimica Acta Part A: Molecular and Biomolecular Spectroscopy 61 (2005) 749-754.

[13] J.M. Hanchar, in:, Uranium $\square$ : Mineralogy, Geochemistry and the Environment, Mineralogical Society of America, 1999.

[14] J. Workman, L. Weyer, Practical Guide to Interpretive Near-infrared Spectroscopy, Taylor \& Francis, 2008.

[15] M.A. Carrell, D.R. Wilder, Journal of Nuclear Materials 13 (1964) 142-151.

[16] A.F. Eidson, Analytical Chemistry 57 (1985) 2134-2138. 


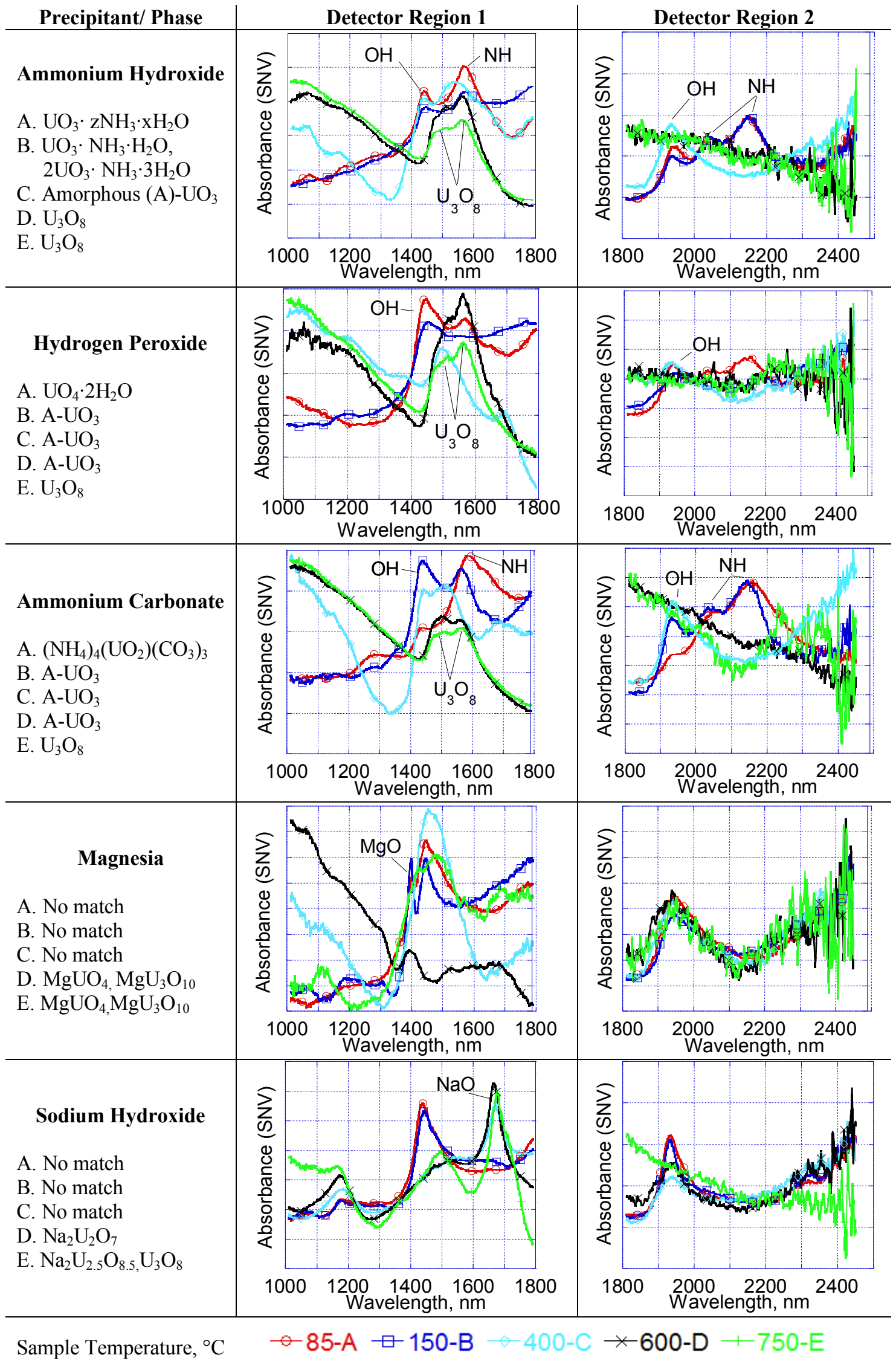

Figure 1: Matrix of NIR spectra and chemical phase information for laboratory-derived precipitates as a function of heat treatment. 


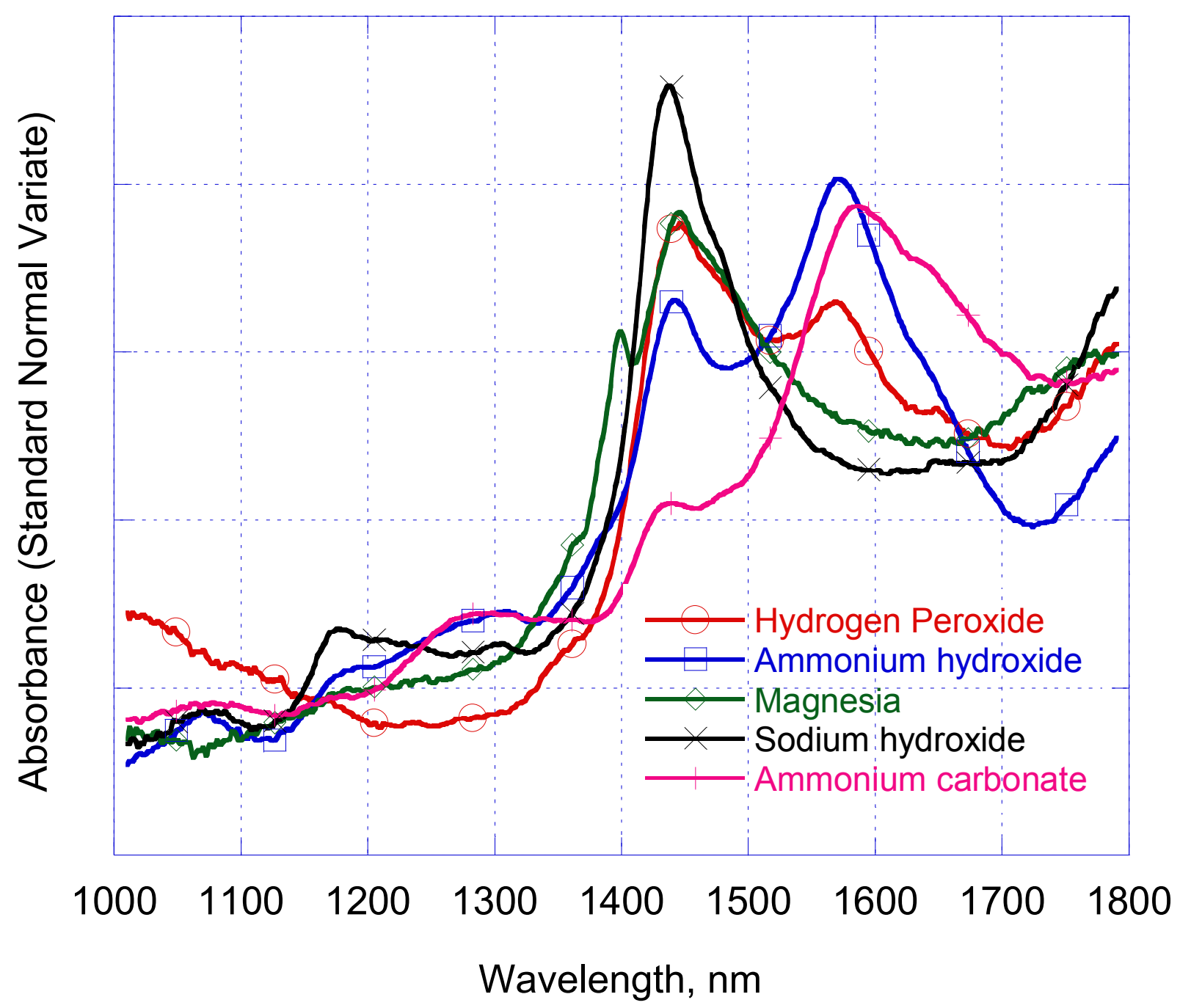

Figure 2: Comparison of NIR spectra for laboratory-derived precipitates heated to $85^{\circ} \mathrm{C}$ 
(a)

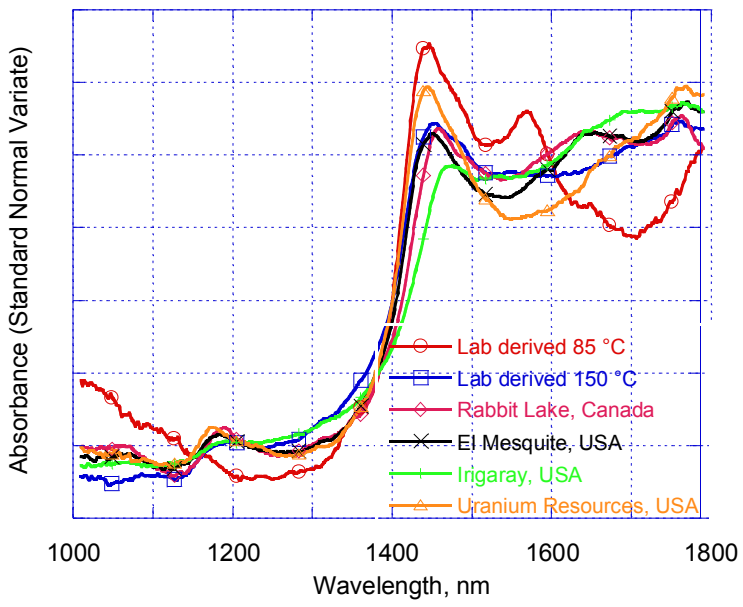

(b)

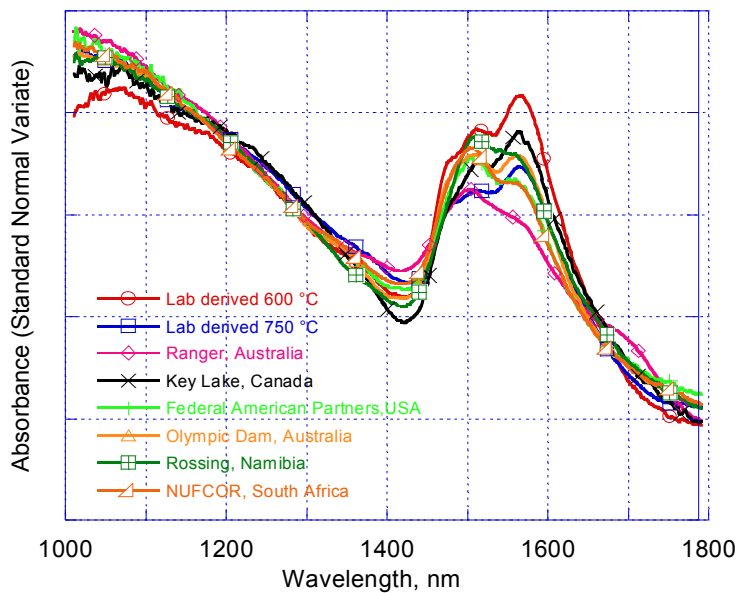

Figure 3: Comparison of NIR spectra for laboratory-derived and real world samples for (a) hydrogen peroxide precipitates heated to low temperatures, all determined to be metastudite $\left(\mathrm{UO}_{4} \cdot 2 \mathrm{H}_{2} \mathrm{O}\right)$ by $\mathrm{x}$-ray diffraction and (b) ammonium hydroxide precipitates heated at elevated temperatures, all determined to be triuranium octooxide $\left(\mathrm{U}_{3} \mathrm{O}_{8}\right)$. 